\title{
Differences in the phenolic composition and nutraceutical properties of freeze dried and oven-dried wild and domesticated samples of Sanguisorba minor Scop
}

\author{
Costanza Ceccanti $^{\text {a,b }}$, Tiane C. Finimundy ${ }^{a}$, Sandrina A. Heleno ${ }^{\text {a, }}{ }^{* *}$, Tania C.S.P. Pires ${ }^{a}$, \\ Ricardo C. Calhelha ${ }^{a}$, Lucia Guidi ${ }^{\text {b, }}$, Isabel C.F.R. Ferreira ${ }^{a}$, Lillian Barros ${ }^{a, *}$ \\ ${ }^{a}$ Centro de Investigação de Montanha (CIMO), Instituto Politécnico de Bragança, Campus de Santa Apolónia, 5300-253, Bragança, Portugal \\ ${ }^{\mathrm{b}}$ Department of Agriculture, Food and Environment, University of Pisa, Via del Borghetto, 80 -56124, Pisa, Italy \\ c Interdepartmental Research Center Nutrafood "Nutraceuticals and Food for Health", University of Pisa, Via del Borghetto, 80, 56124, Pisa, Italy
}

\section{A R T I C L E I N F O}

\section{Keywords:}

Sanguisorba minor

Domestication methods

Chemical characterization

Bioactivities

Drying methods

\begin{abstract}
A B S T R A C T
Recent studies have dealt about the phenolic compounds and the nutraceutical properties of Sanguisorba minor Scop and about the possibility of their domestication to standardise the plant production. However, it is also known that the storage conditions can affect the bioactive compounds present in plants. Thus, wild (W) and domesticated (F1, F2, F3) S. minor samples were exposed to different drying methods (oven-drying at $60^{\circ} \mathrm{C}$ until constant weight named OD or freeze-drying until constant weight named FD) and studied for their content in phenolic compounds, antioxidant, antimicrobial, cytotoxic and anti-inflammatory properties. In general, OD samples showed the highest nutraceutical properties and the highest content in phenolic compounds. The most abundant phenolic compounds in both drying methods were kaempferol-3-O-glucoside and caffeoyl ester, with some differences between wild and domesticated samples. Wild samples showed higher antimicrobial and cytotoxic activity than domesticated ones, except for the OD F3 and FD F2 samples that reported cytotoxicity against HeLa cells. This study provides important information to choose the most adequate methodology to retain phenolic compounds and nutraceutical properties of $S$. minor species. Further researches are necessary to standardise the domestication of the studied wild species and verify the highest efficiency of the OD method.
\end{abstract}

\section{Introduction}

Nowadays, the research of a dietary vegetable variety is increasing, especially in developed countries, to overcome the nutritional deficiencies in the human diet caused by the imposition of using common nutraceutical ingredients derived from the modernisation and globalisation of agriculture (Welch \& Graham, 1999). For this reason, the domestication of wild edible herbs has been studied. Different growing techniques have been applied to wild edible species, in order to evaluate their response to domestication through standard farming systems. These results were significant in terms of nutraceutical properties and phenolic compound content as well as in terms of availability of plant material for an yearly marketability (Ceccanti et al., 2019; Finimundy et al., 2020; Karkanis et al., 2019).

Recently, the consumers, as well as the food industry, have started to concern about the quality of foodstuff. They are demanding for high functional formulations, preferentially with natural and nutraceutical ingredients. Since the use of nutraceutical ingredients is continuously increasing, the drying method as a storage method is resulting of extreme importance in the quality of these ingredients and in the quality of the final food product. Indeed, the retention of bioactive molecules and nutraceutical properties during the use of drying methods is the main concept to have new functional ingredients from wild edible species. It has been noted that the drying methods can inhibit the enzymatic activity, which can lead to the senescence of plant material over variable periods and to the loss of phenolic compounds and nutraceutical properties (Roshanak et al., 2016; Ye et al., 2019). The drying method is considered the oldest food storage technique; thus, different drying methods have been developed to assure the retention of the food constituents (Harbourne et al., 2009). In this perspective, scientific research

\footnotetext{
* Corresponding author.

$* *$ Corresponding author.

E-mail addresses: sheleno@ipb.pt (S.A. Heleno), lillian@ipb.pt (L. Barros).
} 
has been conducted on different wild edible plants, including green tea (Roshanak et al., 2016), Anoectochilus roxburghii (Ye et al., 2019) and Dendrobium nobile (Meng et al., 2018), aiming at analysing the effects of different drying methods. The authors concluded that depending on the drying method, the content in phenolic compounds can be compromised as also the bioactive potential (Meng et al., 2018; Roshanak et al., 2016; Ye et al., 2019).

Ye et al. (2019) observed that vacuum and freeze-drying methods maintained the appearance and the nutraceutical properties of the analysed plant.

In contrast, the hot air and microwave drying methods led to higher retention of polysaccharides. Meng et al. (2018) confirmed these results, showing that the hot air dying method maintained the polysaccharide content, whilst the freeze-drying method led to higher retention of bioactive compounds and antioxidant activity than the hot-air drying method. Conversely, Roshanak et al. (2016) reported that the oven drying method at $60^{\circ} \mathrm{C}$ maintained the antioxidant activity and the total phenolic and flavonoid content, whilst the freeze-drying method maintained the vitamin $\mathrm{C}$ and chlorophyll content.

Sanguisorba minor Scop. is an evergreen perennial species belonging to the Rosaceae family, native from Europe, western Asia and Siberia, and northern Africa (Karkanis et al., 2014). During the ancient times, in famine periods, this species was included in the dishes, mainly in mixtures and soups, and more recently, this herb has been introduced in traditional and folk recipes (Guarrera and Savo 2013, 2016). Nowadays, this species is also considered as a nutraceutical and functional species with health-promoting effects to the human health (Ceccanti et al., 2019; Finimundy et al., 2020; Karkanis et al., 2019). Some studies stated that $S$. minor had interesting nutraceutical properties such as anti-inflammatory activity in over-producing ROS (Radical $\mathrm{O}_{2}$ Species) processes (Kaufmann et al., 2009). This species owned a significant cytotoxic activity against common cancer cell lines, primarily hepatocellular carcinoma line (HepG2), and also antibacterial activity against Bacillus cereus and Staphylococcus aureus (Karkanis et al., 2019). These nutraceutical properties were directly related to the presence of high amounts in phenolic compounds, responsible for the antioxidant activity and, consequently, for cytotoxic, anti-inflammatory and antibacterial activities showed by $S$. minor species (Ceccanti et al., 2019; Ranfa et al., 2014; Vanzani et al., 2011). Thus, a great variety of different phenolic compounds, especially flavonoids, phenolic acids and phenolic alkaloids, such as avenanthramide $2 \mathrm{p}$ and 3,4-diferuloylquinic acid as well as digalloyl-glucoside, catechin, quercetin-O-glucuronide were identified in the aerial parts of this species (Ceccanti et al., 2019; Karkanis et al., 2019).

Given the importance that new wild edible species could have in human health and the introduction of a new source of bioactive compounds and nutraceutical properties in the current monotonous diet, the present work aimed at comparing the nutraceutical properties (i.e., inhibition of lipid peroxidation, antibacterial, anti-inflammatory and cytotoxic properties in a range of tumor cell lines) and the phenolic compound's composition of freeze-dried and oven-dried S. minor plants collected as wild or domesticated by using three different growing systems as detailed in Table 1 (F1, F2, F3) that were analysed separately. Indeed, the retention of phenolic compounds in $S$. minor resulted in fundamental importance in introducing this species as a new functional ingredient in typical food such as pasta or bread.

\section{Material and methods}

\subsection{Plant material}

Wild plant material (W) (about twenty plants) was collected in the Pisa area (Italy, $43^{\circ} 44^{\prime} 39.6^{\prime \prime} \mathrm{N} 10^{\circ} 31^{\prime} 53.39^{\prime \prime} \mathrm{E}$ ) during spring 2019 (April-May), selecting leaves and stems. Regarding the domesticated S. minor samples, the plant material was provided by Tirrenofruit S.r.l (Florence, Italy). This local wholesaler selected three local farms (F1, F2, and F3) for providing the material to be used for the present experiment. The $S$. minor plants were domesticated and sampled according to Ceccanti et al. (2020) and as reported in Table 1 and in Supplementary material (Table S1). The selected farms transferred their consolidated growing systems to the cultivation of $S$. minor plants. $S$. minor young aerial parts, selecting only leaves and stems from twenty plants cultivated in five plots (five plants each plot), were randomly collected in each different farm.

Both wild and domesticated samples were well homogeneized and split into two uniform amounts.

\subsection{Drying processes}

\subsubsection{Oven-drying $(O D)$ and freeze-drying (FD)}

The OD samples were dried in an electric thermostatic oven (Memmert GmbH + Co. KG Universal Oven UN30, Schwabach, Germany) at $60{ }^{\circ} \mathrm{C}$ until it reached a constant weight. The FD samples were dried until it reached the constant weight in a freeze dryer characterised by a standard unheated chamber ( $\varnothing 215 \mathrm{~mm} \times 300 \mathrm{~mm}$ ) (Freeze Dryer Telstar LyoQuest-55, Milan, Italy) through a final condenser temperature of $-55^{\circ} \mathrm{C}$.

\subsection{Extract preparation}

After the applied drying methods, the plant material was ground at $20 \mu \mathrm{m}$ filters and kept in a desiccator protected from light and humidity. The extraction procedure for the determination of the nutraceutical properties and the phenolic compounds was performed according to Bessada et al. (2016). An amount (1 g) of the dried powder of each drying method was stirred with $30 \mathrm{~mL}$ of methanol-water solution $(80: 20, v / v)$ for $60 \mathrm{~min}$ at room temperature. The obtained extracts were filtered through Whatman paper No. 4 filters and evaporated under reduced pressure (Büchi R-210, rotary evaporator, Flawil, Switzerland) at $40{ }^{\circ} \mathrm{C}$ and $100 \mathrm{rpm}$ until methanol was entirely removed. Then, the residual aqueous phase of each sample was frozen, lyophilised (Freezone 4.5, Labconco, Kansas City, MO, USA), and stored in a dry room until further analyses.

Table 1

Details of the three domestications (F1, F2, F3).

\begin{tabular}{|c|c|c|c|c|c|c|c|}
\hline $\begin{array}{l}\text { Domestication } \\
\text { method }\end{array}$ & $\begin{array}{l}\text { Growing Area/ } \\
\text { Location }\end{array}$ & $\begin{array}{l}\text { Duration } \\
\text { Growing Cycle }\end{array}$ & Soil/Substrate & $\begin{array}{l}\text { Pre-treatment of soil/ } \\
\text { Fertigation }\end{array}$ & $\begin{array}{l}\text { Average } \\
\text { Temperature }\end{array}$ & $\begin{array}{l}\text { Average } \\
\text { Relative } \\
\text { Humidity }\end{array}$ & $\begin{array}{l}\text { Daily Mean Solar } \\
\text { Irradiation }\end{array}$ \\
\hline F1 & $\begin{array}{l}\text { Greenhouse } \\
\text { Pisa, Italy }\end{array}$ & 35 days $^{1}$ & Peat & Nutritive solution ${ }^{2}$ & $15.9^{\circ} \mathrm{C}$ & $79.6 \%$ & $287.5 \mathrm{~W} \mathrm{~m}^{-2}$ \\
\hline F2 & $\begin{array}{l}\text { Greenhouse } \\
\text { Siena, Italy }\end{array}$ & 36 days $^{1}$ & Peat & Nutritive solution ${ }^{2}$ & $23.9^{\circ} \mathrm{C}$ & $52.1 \%$ & $365 \mathrm{~W} \mathrm{~m}^{-2}$ \\
\hline F3 & Siena, Italy & One month ${ }^{1}$ & $\begin{array}{l}\text { Mainly silt soil ( } 31 \% \text { clay, } 24 \% \\
\text { sand, } 45 \% \text { silt, } 2 \% \text { organic } \\
\text { matter). }\end{array}$ & $\begin{array}{l}\text { Manure }\left(30 \mathrm{~kg} \mathrm{ha}^{-1}\right) \text { as } \\
\text { pre-treatment of soil, }\end{array}$ & $24.5^{\circ} \mathrm{C}$ & $46.0 \%$ & $1050 \mathrm{~W} \mathrm{~m}^{-2}$ \\
\hline
\end{tabular}

$\overline{1,2}$ Adapted of Ceccanti et al. (2020) and reported in Supplementary material (Table S1). 


\subsection{Determination of phenolic compounds}

The phenolic compounds were determined according to Bessada et al. (2016). The extracted samples were dissolved in methanol-water solution $(20: 80 \mathrm{v} / \mathrm{v})$ to a final concentration of $10 \mathrm{mg} / \mathrm{mL}$ and filtered through a $0.22 \mu \mathrm{m}$ disposable filters. A Dionex Ultimate 3000 (Dionex Ultimate 3000 UPLC and Linear Ion Trap LQT XL, Thermo Scientific, San Jose, CA, USA) ultra-performance liquid chromatographic equipment coupled to a diode array detector and an electrospray ionisation mass spectrometry detector (HPLC-DAD-ESI/MS) was used to analyse the samples. The acquisition and the processing of data were carried out with the Xcalibur ${ }^{\circledR}$ data system (Thermo Scientific, San Jose, CA, USA). Authentic standards (Extrasynthése S.A., Genay, France) and data available from the literature were used to identify individual phenolic compounds. The quantification of the detected compounds was based on the calibration curves of authentic standards. The results were presented as $\mathrm{mg} / \mathrm{g}$ extract.

\subsection{Nutraceutical properties}

\subsubsection{Antioxidant activity}

The antioxidant activity was evaluated by the inhibition of lipid peroxidation using thiobarbituric acid reactive substances (TBARS). The obtained extracts were dissolved in a methanol solution $(80: 20 \mathrm{v} / \mathrm{v})$ to obtain a stock solution of $5 \mathrm{mg} / \mathrm{mL}$. Afterward, successive dilutions were made to obtain a range of concentrations of 40 to $0.3 \mu \mathrm{g} / \mathrm{mL}$. Briefly, porcine (Sus scrofa) brains were homogenized in ice-cold Tris-HCl buffer $(20 \mathrm{~mm} / \mathrm{L}, \mathrm{pH} 7.4)$. An aliquot $(0.1 \mathrm{~mL})$ of the supernatant was incubated with the different solution concentrations $(0.2 \mathrm{~mL})$ in the presence of $\mathrm{FeSO}_{4}(10 \mu \mathrm{m} / \mathrm{L} ; 0.1 \mathrm{~mL})$ and ascorbic acid $(0.1 \mathrm{~mm} / \mathrm{L} ; 0.1 \mathrm{~mL})$ at $37^{\circ} \mathrm{C}$ for $1 \mathrm{~h}$. The reaction was stopped by the addition of trichloroacetic acid $(28 \% \mathrm{w} / \mathrm{v}, 0.5 \mathrm{~mL})$, followed by thiobarbituric acid (TBA, $2 \%, \mathrm{w} / \mathrm{v}$, $0.38 \mathrm{~mL}$ ), and the mixture was then heated at $80{ }^{\circ} \mathrm{C}$ for $20 \mathrm{~min}$. The antioxidant activity resulted from the reduction of TBARS, resulting in the formation of the malondialdehyde-thiobarbituric acid complex (MDA-TBA). The colour intensity, displayed by this complex in the different working concentrations, was measured by its absorbance at $532 \mathrm{~nm}$ (UV-Vis Specord 200 spectrophotometer, Analytik Jena). The results were expressed in values of $\mathrm{EC}_{50}$, the necessary concentration of sample able to obtain half of antioxidant activity (Mandim et al., 2020).

\subsubsection{Antibacterial activity}

The bacterial strains were isolated from patients hospitalised in various departments at the North-eastern local health unit (Bragança, Portugal) and Hospital Center of Trás-os-Montes and Alto Douro (Vila Real, Portugal). Five Gram-negative bacteria: Escherichia coli (isolated from urine), Proteus mirabilis (isolated from wound exudate), Klebsiella pneumoniae (isolated from urine), Pseudomonas aeruginosa (isolated from expectoration) and Morganella morganii (isolated from urine); and three Gram-positive bacteria: Enterococcus faecalis (isolated from urine), Listeria Monocytogenes (isolated from cerebrospinal fluid), and methicillinresistant $S$. aureus (MRSA) (isolated from expectoration) were tested. All these microorganisms were incubated at $37{ }^{\circ} \mathrm{C}$ in an appropriate fresh medium for $24 \mathrm{~h}$ before analysis to achieve the exponential growth phase.

The determination of the Minimal Inhibitory Concentration (MIC) was conducted by the microdilution method and the colourimetric method using $p$-iodonitrotetrazolium chloride (INT) (Panreac Applichem-Barcelona, Spain), according to Pires et al. (2018). Briefly, the extracted samples were dissolved in $5 \mathrm{~mL} / \mathrm{mL}(v / v)$ dimethyl sulfoxide (DMSO) and $95 \mathrm{~mL}$ of Tryptic Soy Broth (TSB) to a final concentration of $20 \mathrm{mg} / \mathrm{mL}$. Afterward, successive dilutions were performed in the 96 well microplates, testing a range of concentrations between 20 and $0.15 \mathrm{mg} / \mathrm{mL}$. Finally, $10 \mu \mathrm{L}$ of inoculum [standardised at $1.5 \times 10^{8}$ Colony Forming Unit (CFU)/mL] were added to all the microplate wells. Three negative controls (one with TSB, another one with the extract, and the third with medium, antibiotics, and bacteria), and one positive control was prepared with Mueller-Hinton broth (MHB), TSB and each inoculum. Ampicillin and imipenem were used for all the Gram-negative bacteria and $L$. monocytogenes, while ampicillin and vancomycin were selected for $E$. faecalis and MRSA. After an incubation period at $37{ }^{\circ} \mathrm{C}$ for $24 \mathrm{~h}$, the MIC was detected after the addition of INT and incubation at $37{ }^{\circ} \mathrm{C}$ for $30 \mathrm{~min}$. MIC was defined as the lowest concentration that inhibits the visible bacterial growth determined by the change of the colour from yellow to pink in viable microorganisms. For the determination of the Minimum Bactericidal Concentration (MBC), the lowest concentration allowing to kill the bacterial strains, $10 \mu \mathrm{L}$ of liquid from each well, that showed no change in colour in the previous test, were plated on solid medium, i.e., blood agar $(0.07 \mathrm{~mL}$ sheep blood) and incubated at $37^{\circ} \mathrm{C}$ for $24 \mathrm{~h}$. The lowest concentration that yielded no growth determined the MBC.

\subsubsection{Cytotoxic activity}

The cytotoxic activity was evaluated in four different human tumour cell lines obtained from DSMZ- Leibniz - Institut DSMZ - Deutsche Sammlung von Mikroorganismen und Zellkulturen (HeLa: cervical carcinoma; HepG2: hepatocellular carcinoma; MCF-7: breast adenocarcinoma; and NCI-H460: non-small-cell lung cancer) using the sulphorhodamine B (SRB) assay as described by Guimarães et al. (2013). RPMI-1640 medium containing $0.1 \mathrm{~mL}$ heat-inactivated FBS and 2 $\mathrm{mmol} / \mathrm{L}$ glutamine was used to routinely maintain the adherent cell cultures at $37{ }^{\circ} \mathrm{C}$, in a humidified air incubator containing $\mathrm{CO}_{2}$. Additionally, a primary cellular line from the porcine liver (PLP) was used as a normal cell line to achieve the extract's toxicity. Each cell line was plated at an appropriate density $\left(7.5 \times 10^{3}\right.$ cells/well for MCF-7, NCI-H460 and HCT-15 or $1.0 \times 10^{4}$ cells/well for HeLa and HepG2) in 96-well plates and allowed to attach for $24 \mathrm{~h}$. Then, cells were treated for $48 \mathrm{~h}$ with different concentrations of the extracts in a range of 400 to $1.56 \mu \mathrm{g} / \mathrm{mL}$.

The adherent cells were fixed with trichloroacetic acid, incubated for $60 \mathrm{~min}$ at $4{ }^{\circ} \mathrm{C}$, washed with deionised water and dried. After this process, SRB $(0.001 \mu \mathrm{L}$ in $0.01 \mu \mathrm{L}$ acetic acid, $100 \mu \mathrm{L})$ was added to the microplate wells and incubated for $30 \mathrm{~min}$ at room temperature.

The adherent SRB was solubilised with Tris $(200 \mu \mathrm{L})$, and the absorbance was read at $540 \mathrm{~nm}$ in the microplate reader (ELX800 Biotek). The anti-proliferative activity was assessed as the extract concentration providing half of cell growth inhibition $\left(\mathrm{GI}_{50}, \mu \mathrm{g} / \mathrm{mL}\right)$. Ellipticin was used as a positive control.

\subsubsection{Anti-inflammatory activity}

The anti-inflammatory activity was assessed following a procedure described by Svobodova et al. (2017). The dried extracts were evaluated in mouse lipopolysaccharide (LPS)-stimulated macrophage-like cell line RAW 264.7 in a concentration range from 400 to $1.56 \mu \mathrm{g} / \mathrm{mL}$. Aliquots of 150,000 cells/well were seeded in 96-well plates, and the cells were left to attach to the plate overnight. Then, cells were treated with prepared concentrations of the sample extracts for $1 \mathrm{~h}$. Further, the cells were stimulated with LPS $(1 \mu \mathrm{g} / \mathrm{mL})$ for $18 \mathrm{~h}$. Dexamethasone $(50$ $\mu \mathrm{mol} / \mathrm{L}$ ) was used as a positive control for the experiment. The effect of all analysed samples in the absence of LPS was also evaluated in order to observe if they induced chances in nitric oxide basal levels. In negative controls, no LPS was added. Both extracts and LPS were dissolved in DMEM cell culture medium supplemented with $0.1 \mathrm{~mL}$ heat-inactivated fetal bovine serum, glutamine, and antibiotics. For the determination of nitric oxide, a Griess Reagent System kit was used, which contains sulphanilamide, N-(1-naphtyl)ethylenediamine hydrochloride (NED), and nitrite solutions. A reference standard curve of nitrite was prepared in a 96-well plate. The cell culture supernatant was transferred to the plate and mixed with sulphanilamide and NED solutions at room temperature. The production of nitric oxide was determined by measuring the absorbance at $540 \mathrm{~nm}$ in the microplate reader (ELX800 Biotek) and by comparison with the standard calibration curve. The results were 
expressed as $\mathrm{EC}_{50}$ values $(\mu \mathrm{g} / \mathrm{mL}$ ) (sample concentration providing half of anti-inflammatory activity and, thus, half-inhibition of nitric oxide), and dexamethasone $(50 \mu \mathrm{mol} / \mathrm{L})$ was used as a positive control.

\subsection{Statistical analysis}

The experimental layout was arranged according to the completely randomized design. The statistical analysis was carried out by using SPSS v. 23.0 software and using the one-way analysis of variance (ANOVA), while means were compared with Tukey's HSD test $(p<$
0.05). Three samples were analysed for each treatment, and all the assays were carried out in triplicate. Data were expressed as mean \pm standard deviation.

\section{Results and discussion}

\subsection{Phenolic composition}

Data (retention time, $\lambda_{\max }$ in the visible region, molecular ion, and main fragment ions observed in $\mathrm{MS}^{2}$ ) obtained by HPLC-DAD-ESI/MS

Table 2

Identification and quantification of phenolic compounds in $S$. minor aerial parts.

\begin{tabular}{|c|c|c|c|c|c|c|c|c|c|c|c|c|c|}
\hline \multirow[t]{3}{*}{ Peaks } & \multirow{3}{*}{$\begin{array}{l}\text { Rt } \\
(\min )\end{array}$} & \multirow{3}{*}{$\begin{array}{l}\lambda_{\max } \\
(\mathrm{nm})\end{array}$} & \multirow{3}{*}{$\begin{array}{l}\text { Molecular } \\
\text { ion }[\mathrm{M}-\mathrm{H}]^{-} \\
(\mathrm{m} / \mathrm{z})\end{array}$} & \multirow[t]{3}{*}{$\mathrm{MS}^{2}(\mathrm{~m} / \mathrm{z})$} & \multirow{3}{*}{$\begin{array}{l}\text { Tentative } \\
\text { identification }\end{array}$} & \multicolumn{8}{|c|}{ Quantification (mg/g) } \\
\hline & & & & & & \multicolumn{4}{|c|}{ Oven-dried } & \multicolumn{4}{|c|}{ Freeze-dried } \\
\hline & & & & & & $\mathrm{W}$ & F1 & F2 & F3 & $\mathrm{W}$ & $\mathrm{F} 1$ & $\mathrm{~F} 2$ & F3 \\
\hline 1 & 3.87 & 267 & 331 & $\begin{array}{l}271(35), 167 \\
(100)\end{array}$ & $\begin{array}{l}\text { Gallic acid } \\
\text { glucoside }^{\mathrm{a}}\end{array}$ & $26 \pm 2 a$ & $\begin{array}{l}0.64 \pm \\
0.02 \mathrm{e}\end{array}$ & $\begin{array}{l}1.6846 \\
\pm \\
0.0004 d\end{array}$ & $\begin{array}{l}2.40 \pm \\
0.01 \mathrm{~cd}\end{array}$ & $\begin{array}{l}3.73 \pm \\
0.02 b\end{array}$ & $\begin{array}{l}0.63 \pm \\
0.02 \mathrm{e}\end{array}$ & $\begin{array}{l}2.85 \pm \\
0.05 c\end{array}$ & $\begin{array}{l}3.11 \pm \\
0.01 \mathrm{bc}\end{array}$ \\
\hline 2 & 4.53 & 323 & 353 & $\begin{array}{l}\text { 191(100), } \\
179(44)\end{array}$ & $\begin{array}{l}\text { 3-O- } \\
\text { Caffeoylquinic } \\
\operatorname{acid}^{\mathrm{b}}\end{array}$ & $22 \pm 1 \mathrm{a}$ & $\begin{array}{l}0.81 \pm \\
0.01 \mathrm{fg}\end{array}$ & $\begin{array}{l}1.48 \pm \\
0.03 \mathrm{e}\end{array}$ & $\begin{array}{l}3.689 \pm \\
0.004 c\end{array}$ & $\begin{array}{l}12.01 \\
\pm \\
0.04 \mathrm{~b}\end{array}$ & $\begin{array}{l}0.69 \pm \\
0.01 g\end{array}$ & $\begin{array}{l}1.329 \pm \\
0.001 \mathrm{ef}\end{array}$ & $\begin{array}{l}3.0 \pm \\
0.1 \mathrm{~d}\end{array}$ \\
\hline 3 & 5.29 & 321 & 595 & $\begin{array}{l}297(5), 179 \\
(34), 135 \\
(100)\end{array}$ & 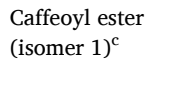 & $\begin{array}{l}14.8 \pm \\
0.1 \mathrm{a}\end{array}$ & $\begin{array}{l}0.87 \pm \\
0.02 g\end{array}$ & $\begin{array}{l}7.6 \pm \\
0.1 \mathrm{e}\end{array}$ & $\begin{array}{l}11.0 \pm \\
0.2 \mathrm{c}\end{array}$ & $\begin{array}{l}12.38 \\
\pm \\
0.02 \mathrm{~b}\end{array}$ & $\begin{array}{l}0.5 \pm \\
0.02 \mathrm{~h}\end{array}$ & $\begin{array}{l}2.91 \pm \\
0.05 f\end{array}$ & $\begin{array}{l}8.9 \pm \\
0.1 \mathrm{~d}\end{array}$ \\
\hline 4 & 6.79 & 286 & 483 & $313(100)$ & $\begin{array}{l}\text { Digalloyl } \\
\text { glucoside }^{\mathrm{d}}\end{array}$ & $22 \pm 1 a$ & $\begin{array}{l}0.94 \pm \\
0.02 \mathrm{fg}\end{array}$ & $\begin{array}{l}5.0 \pm \\
0.2 \mathrm{~d}\end{array}$ & $\begin{array}{l}4.8 \pm \\
0.2 \mathrm{~d}\end{array}$ & $\begin{array}{l}5.6 \pm \\
0.3 \mathrm{~b}\end{array}$ & $\begin{array}{l}0.365 \\
\pm \\
0.003 \mathrm{~g}\end{array}$ & $\begin{array}{l}1.49 \pm \\
0.02 \mathrm{f}\end{array}$ & $\begin{array}{l}3.5 \pm \\
0.1 \mathrm{e}\end{array}$ \\
\hline 5 & 8.34 & 321 & 595 & $\begin{array}{l}297(5), 179 \\
(34), 135 \\
(100)\end{array}$ & 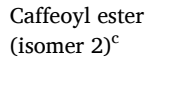 & $\begin{array}{l}5.7 \pm \\
0.2 \mathrm{a}\end{array}$ & $\begin{array}{l}0.17 \pm \\
0.03 f\end{array}$ & $\begin{array}{l}1.47 \pm \\
0.01 \mathrm{~d}\end{array}$ & $\begin{array}{l}3.35 \pm \\
0.02 b\end{array}$ & $\begin{array}{l}1.16 \pm \\
0.01 \mathrm{e}\end{array}$ & $\begin{array}{l}0.078 \\
\pm \\
0.004 \mathrm{~g}\end{array}$ & $\begin{array}{l}1.19 \pm \\
0.02 \mathrm{e}\end{array}$ & $\begin{array}{l}2.26 \pm \\
0.01 \mathrm{c}\end{array}$ \\
\hline 6 & 12.79 & 228 & 1401 & $\begin{array}{l}935(57), 897 \\
(), 633(10)\end{array}$ & Lambertianin $C^{d}$ & $\begin{array}{l}18.6 \pm \\
0.3 a\end{array}$ & $\begin{array}{l}1.76 \pm \\
0.02 \mathrm{~d}\end{array}$ & $\begin{array}{l}9.7 \pm \\
0.4 \mathrm{~b}\end{array}$ & $\begin{array}{l}5.3 \pm \\
0.1 c\end{array}$ & nd & $\begin{array}{l}1.401 \\
\pm \\
0.002 \mathrm{e}\end{array}$ & $\begin{array}{l}5.31 \pm \\
0.03 c\end{array}$ & $\begin{array}{l}1.87 \pm \\
0.02 \mathrm{~d}\end{array}$ \\
\hline 7 & 13.43 & 241 & 934 & $\begin{array}{l}\text { 633(100), } \\
301(56)\end{array}$ & $\begin{array}{l}\text { Galloyl-bis- } \\
\text { HHDP-glucoside }^{\mathrm{d}}\end{array}$ & $\begin{array}{l}5.5 \pm \\
0.1 \mathrm{a}\end{array}$ & $\begin{array}{l}1.8 \pm \\
0.1 \mathrm{e}\end{array}$ & $\begin{array}{l}1.93 \pm \\
0.03 \mathrm{~d}\end{array}$ & $\begin{array}{l}2.03 \pm \\
0.02 c\end{array}$ & $\begin{array}{l}1.89 \pm \\
0.02 \mathrm{~d}\end{array}$ & $\begin{array}{l}1.46 \pm \\
0.01 \mathrm{f}\end{array}$ & $\begin{array}{l}1.54 \pm \\
0.02 \mathrm{f}\end{array}$ & $\begin{array}{l}2.75 \pm \\
0.05 b\end{array}$ \\
\hline 8 & 15.03 & 222 & 1567 & $\begin{array}{l}1265(100), \\
1103(8), 933 \\
(16), 783(5), \\
633(77), 301 \\
(65)\end{array}$ & Sanguin $\mathrm{H}-10^{\mathrm{d}}$ & $\begin{array}{l}18.3 \pm \\
0.4 \mathrm{a}\end{array}$ & $\begin{array}{l}1.31 \pm \\
0.01 \mathrm{~d}\end{array}$ & $\begin{array}{l}3.01 \pm \\
0.06 \mathrm{~b}\end{array}$ & $\begin{array}{l}3.15 \pm \\
0.01 b\end{array}$ & nd & $\begin{array}{l}1.257 \\
\pm \\
0.001 \mathrm{~d}\end{array}$ & $\begin{array}{l}2.57 \pm \\
0.03 c\end{array}$ & nd \\
\hline 9 & 16.32 & 256 & 615 & $\begin{array}{l}463(100) \\
301(10)\end{array}$ & 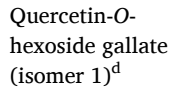 & $\begin{array}{l}4.5 \pm \\
0.1 \mathrm{a}\end{array}$ & $\begin{array}{l}0.60 \pm \\
0.04 g\end{array}$ & $\begin{array}{l}1.65 \pm \\
0.04 \mathrm{~d}\end{array}$ & $\begin{array}{l}2.312 \pm \\
0.001 c\end{array}$ & $\begin{array}{l}0.76 \pm \\
0.01 \mathrm{f}\end{array}$ & $\begin{array}{l}0.57 \pm \\
0.004 \mathrm{~g}\end{array}$ & $\begin{array}{l}1.079 \pm \\
0.002 \mathrm{e}\end{array}$ & $\begin{array}{l}2.64 \pm \\
0.02 b\end{array}$ \\
\hline 10 & 16.67 & 346 & 615 & $\begin{array}{l}463(15), 301 \\
(100)\end{array}$ & 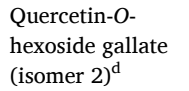 & $\begin{array}{l}5.3 \pm \\
0.2 \mathrm{a}\end{array}$ & $\begin{array}{l}0.784 \\
\pm \\
0.004 \mathrm{e}\end{array}$ & $\begin{array}{l}1.13 \pm \\
0.05 \mathrm{~d}\end{array}$ & $\begin{array}{l}1.81 \pm \\
0.02 b\end{array}$ & $\begin{array}{l}1.27 \pm \\
0.01 \mathrm{c}\end{array}$ & $\begin{array}{l}0.562 \\
\pm \\
0.001 \mathrm{f}\end{array}$ & $\begin{array}{l}0.82 \pm \\
0.01 \mathrm{e}\end{array}$ & nd \\
\hline 11 & 17.75 & 354 & 477 & $301(100)$ & $\begin{array}{l}\text { Quercetin-3-O- } \\
\text { glucuronide }^{\mathrm{e}}\end{array}$ & $\begin{array}{l}18.0 \pm \\
0.1 \mathrm{a}\end{array}$ & $\begin{array}{l}2.61 \pm \\
0.04 \mathrm{f}\end{array}$ & $\begin{array}{l}3.9 \pm \\
0.2 \mathrm{~d}\end{array}$ & $\begin{array}{l}2.30 \pm \\
0.05 \mathrm{~g}\end{array}$ & $\begin{array}{l}5.48 \pm \\
0.01 \mathrm{c}\end{array}$ & $\begin{array}{l}1.23 \pm \\
0.01 \mathrm{~h}\end{array}$ & $\begin{array}{l}3.00 \pm \\
0.02 \mathrm{e}\end{array}$ & $\begin{array}{l}9.42 \pm \\
0.02 b\end{array}$ \\
\hline 12 & 20.42 & 346 & 447 & $285(100)$ & $\begin{array}{l}\text { Kaempferol-3-O- } \\
\text { glucoside }^{\mathrm{e}}\end{array}$ & $\begin{array}{l}11.3 \pm \\
0.6 \mathrm{~b}\end{array}$ & $\begin{array}{l}1.49 \pm \\
0.01 \mathrm{e}\end{array}$ & $\begin{array}{l}1.995 \pm \\
0.003 \mathrm{~d}\end{array}$ & $\begin{array}{l}27.6 \pm \\
0.1^{\circ}\end{array}$ & $\begin{array}{l}5.13 \pm \\
0.03 c\end{array}$ & $\begin{array}{l}0.30 \pm \\
0.01 g\end{array}$ & $\begin{array}{l}0.62 \pm \\
0.01 \mathrm{f}\end{array}$ & nd \\
\hline 13 & 21.42 & 341 & 609 & $301(100)$ & $\begin{array}{l}\text { Quercetin-3-O- } \\
\text { rutinoside }\end{array}$ & nd & $\begin{array}{l}0.53 \pm \\
0.01 \mathrm{e}\end{array}$ & $\begin{array}{l}0.43 \pm \\
0.01 \mathrm{f}\end{array}$ & $\begin{array}{l}2.99 \pm \\
0.01^{\circ}\end{array}$ & nd & $\begin{array}{l}0.58 \pm \\
0.01 \mathrm{~d}\end{array}$ & $\begin{array}{l}1.047 \pm \\
0.005 c\end{array}$ & $\begin{array}{l}2.21 \pm \\
0.03 b\end{array}$ \\
\hline 14 & 21.55 & 344 & 447 & $285(100)$ & $\begin{array}{l}\text { Kaempferol-O- } \\
\text { hexoside }^{\mathrm{e}}\end{array}$ & $\begin{array}{l}9.84 \pm \\
0.04 \mathrm{a}\end{array}$ & nd & nd & nd & $\begin{array}{l}3.5 \pm \\
0.1 \mathrm{~b}\end{array}$ & nd & nd & nd \\
\hline & & & & & $\begin{array}{l}\text { Total phenolic } \\
\text { acids }\end{array}$ & $22 \pm 1 \mathrm{a}$ & $\begin{array}{l}0.81 \pm \\
0.01 \mathrm{fg}\end{array}$ & $\begin{array}{l}1.48 \pm \\
0.03 e\end{array}$ & $\begin{array}{l}3.689 \pm \\
0.004 c\end{array}$ & $\begin{array}{l}12 \pm \\
0.04 \mathrm{~b}\end{array}$ & $\begin{array}{l}0.69 \pm \\
0.01 g\end{array}$ & $\begin{array}{l}1.329 \pm \\
0.001 \mathrm{ef}\end{array}$ & $\begin{array}{l}3.0 \pm \\
0.1 \mathrm{~d}\end{array}$ \\
\hline & & & & & $\begin{array}{l}\text { Total } \\
\text { hydrolysable } \\
\text { tannins }\end{array}$ & $111 \pm 1 \mathrm{a}$ & $\begin{array}{l}7.5 \pm \\
0.1 \mathrm{~g}\end{array}$ & $30 \pm 1 c$ & $\begin{array}{l}31.993 \\
\pm \\
0.001 b\end{array}$ & $\begin{array}{l}24.8 \pm \\
0.3 \mathrm{~d}\end{array}$ & $\begin{array}{l}5.69 \pm \\
0.04 \mathrm{~h}\end{array}$ & $\begin{array}{l}17.8 \pm \\
0.2 f\end{array}$ & $\begin{array}{l}22.4 \pm \\
0.3 e\end{array}$ \\
\hline & & & & & Total flavonoids & $\begin{array}{l}49.0 \pm \\
0.5 \mathrm{a}\end{array}$ & $\begin{array}{l}6 \pm \\
0.1 g\end{array}$ & $\begin{array}{l}9.1 \pm \\
0.2 \mathrm{e}\end{array}$ & $\begin{array}{l}36.97 \pm \\
0.03 \mathrm{~b}\end{array}$ & $\begin{array}{l}16.1 \pm \\
0.2 \mathrm{c}\end{array}$ & $\begin{array}{l}3.20 \pm \\
0.03 \mathrm{~h}\end{array}$ & $\begin{array}{l}6.56 \pm \\
0.05 f\end{array}$ & $\begin{array}{l}14.3 \pm \\
0.1 \mathrm{~d}\end{array}$ \\
\hline & & & & & $\begin{array}{l}\text { Total phenolic } \\
\text { compounds }\end{array}$ & $182 \pm 2 \mathrm{a}$ & $\begin{array}{l}14.3 \pm \\
0.1 \mathrm{~g}\end{array}$ & $41 \pm 1 d$ & $\begin{array}{l}72.65 \pm \\
0.02 \mathrm{~b}\end{array}$ & $\begin{array}{l}52.9 \pm \\
0.3 c\end{array}$ & $\begin{array}{l}9.62 \pm \\
0.1 \mathrm{~h}\end{array}$ & $\begin{array}{l}25.7 \pm \\
0.3 f\end{array}$ & $\begin{array}{l}39.7 \pm \\
0.4 \mathrm{e}\end{array}$ \\
\hline
\end{tabular}

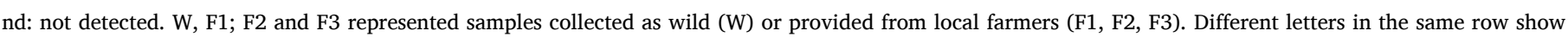

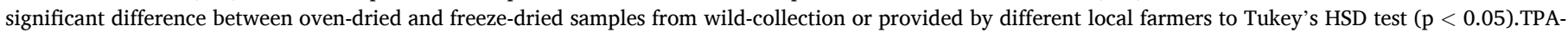

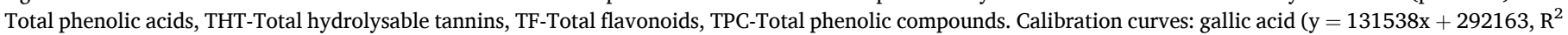

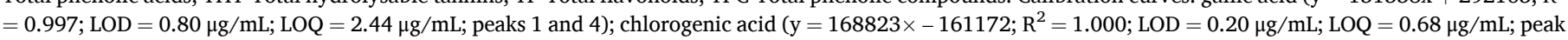

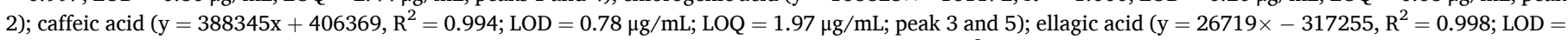

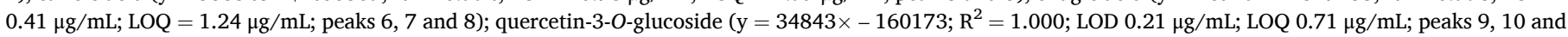

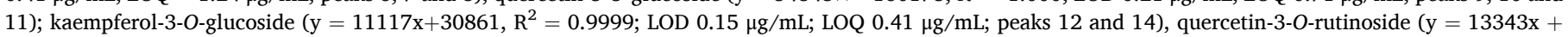

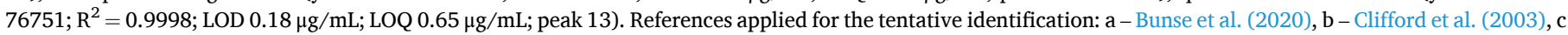
- Parveen et al. (2008), d - Finimundy et al. (2020), and e - standard, DAD and MS fragmentation pattern. 
analysis regarding phenolic compounds identification and individual quantification are presented in Table 2, and a typical chromatogram is presented in Fig. 1. Fourteen phenolic compounds were detected, being one phenolic acid, six flavonoids, and seven hydrolyzable tannins. Compounds 11,12 , and 13 were positively identified as quercetin-3-Oglucuronide, kaempferol-3-O-glucoside, quercetin-3-O-rutinoside, respectively, by comparison with authentic standards and analysis of their MS fragmentation pattern, retention time, and UV-vis characteristics. These compounds were already reported in $S$. minor by other authors (Finimundy et al., 2020; Karkanis et al., 2019). The majority of the identified compounds (peaks 6, 7, 8, and 9) were previously identified by Finimundy et al. (2020) and, therefore, identified in this study considering the previously described molecular ions of the molecules.

Compound $2\left([\mathrm{M}-\mathrm{H}]^{-}\right.$at $\left.\mathrm{m} / \mathrm{z} 353\right)$ was identified as 3-O-caffeoylquinic acid based on the fragmentation pattern reported by Clifford et al. (2003). Peak $1\left([\mathrm{M}-\mathrm{H}]^{-}\right.$at $m / z$ 331) identified as a gallic acid glucoside was recently reported in Sanguisorba officinalis by Bunse et al. (2020). Peaks 3 and 5 presented the same pseudomolecular ion $\left([\mathrm{M}-\mathrm{H}]^{-}\right.$at $m / z$ 595) and fragmentation patterns; they were identified as two isomers and were assigned to caffeoyl ester based on the literature where they were found in orchardgrass (Parveen et al., 2008). Peak $14\left([\mathrm{M}-\mathrm{H}]^{-}\right.$at $\mathrm{m} / \mathrm{z} 447$ ) presented the same characteristics (DAD and fragmentation pattern) as peak 12; nevertheless, no information about the identity of the sugar moiety and the location on the aglycone could be obtained, so the compound 14 was tentatively identified as kaempferol-O-hexoside.

OD samples analysed in the present work exhibited a higher content in total phenolic compounds than FD samples, revealing a higher concentration of phenolic acids, total hydrolyzable tannins, and total flavonoids. As for the qualitative composition, FD samples did not reveal the presence of lambertianin $\mathrm{C}$, sanguiin- $\mathrm{H}$, and quercetin- $O$-hexoside gallate in wild samples. Moreover, both the OD and the FD samples did not present quercetin-O-rutinoside in wild plants and kaempferol-3-Oglucoside in F3 cultivated plants, showing that not only the drying method can affect the phenolic composition but also the different growing technique and the double effect of drying method and different growing technique. However, in this study, the cultivation parameters (temperature, moisture, solar irradiation, and soil or substrates characteristics) were not standardised during the experiment, which must have the main purpose of explaining how different drying methods affect the phytochemical content and the bioactive properties of $S$. minor species. Neverthelss, we considered useful the domestication of a new species as $S$. minor for the future marketability of a relatively known species.

Most studies reported FD as the most efficient drying method because this method leads to the rupture of cell structure caused by ice crystals formed within plant matrix and, consequently, promotes the extraction efficiency in FD samples, retaining the phenolic content (Meng et al., 2018; Pinela et al., 2012). Conversely, other authors described that $60{ }^{\circ} \mathrm{C}$ used in OD is the most indicated strategy in maintaining phytochemical content (Ye et al., 2019; Youssef \& Mokhtar, 2014), which followed the results obtained in the present study. Moreover, the obtained results suggested that phenolic compounds deterioration may be affected by many factors other than temperature treatments. These factors may include the activity of polyphenol oxidase (PPO), drying times, and plant moisture (Youssef \& Mokhtar, 2014). PPO catalyzes the oxidation of phenols into quinones, which subsequently deteriorate and polymerize into brown pigments (Terefe et al., 2010). Celluar destruction due to extensive drying times promotes phenolic compounds' loss by increasing their polarity in water. In this way, water allows phenolic compounds to dissolve, being dragged to the surface (Alean et al., 2016). Moreover, the higher phenolic content of OD samples compared to FD samples could be linked to more effective extraction of the insoluble phenolic compounds such as phenolic acids or condensed tannins linked to cell wall polysaccharides or, more specifically, proteins (Shonte et al., 2020).

\subsection{Antioxidant activity}

S. minor extracts with different origins i.e., collected as wild (W) or cultivated in different local farms (F1, F2, F3) were tested for the capacity to inhibit the lipid peroxidation. Fig. 2 reports results obtained by the TBARS assay.

Comparing OD and FD plant material from the same origin, OD $S$. minor plants revealed the highest lipid peroxidation inhibition by TBARS assay $\left(\mathrm{EC}_{50}\right.$ of OD W, F1, F2, F3 samples $=0.005 \pm 0.0002 ; 0.03$ $\pm 0.002 ; 0.005 \pm 0.0002 ; 0.03 \pm 0.0001 \mu \mathrm{g} / \mathrm{mL}$, respectively). The
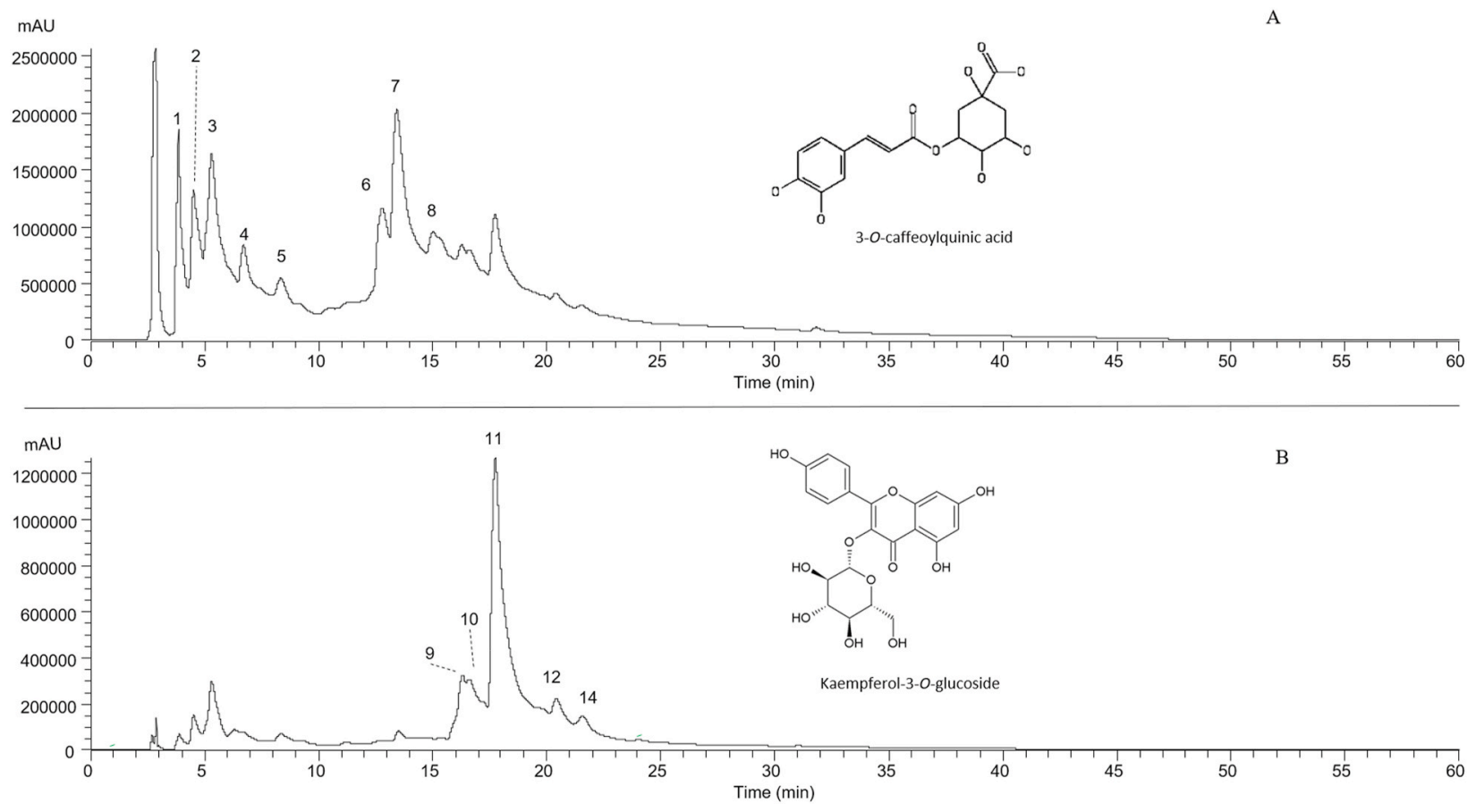

Fig. 1. Phenolic profile of oven-dried S. minor plant collected as wild recorded at $280 \mathrm{~nm}$ (A) and $320 \mathrm{~nm}$ (B). For peak, numbers refer to Table 2. 

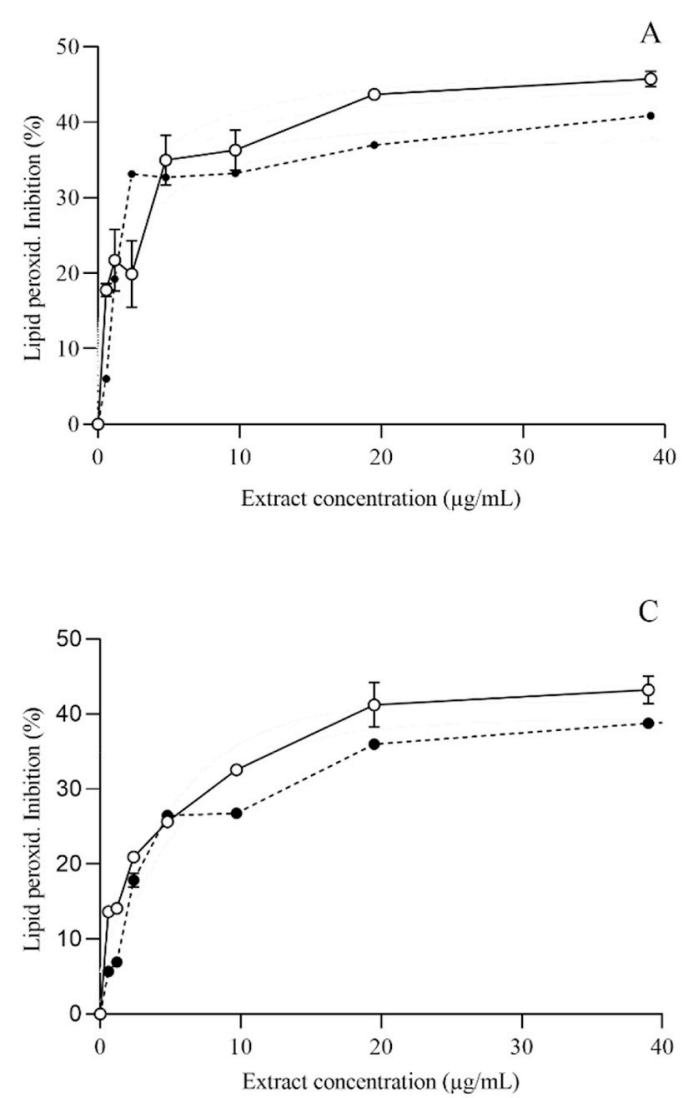

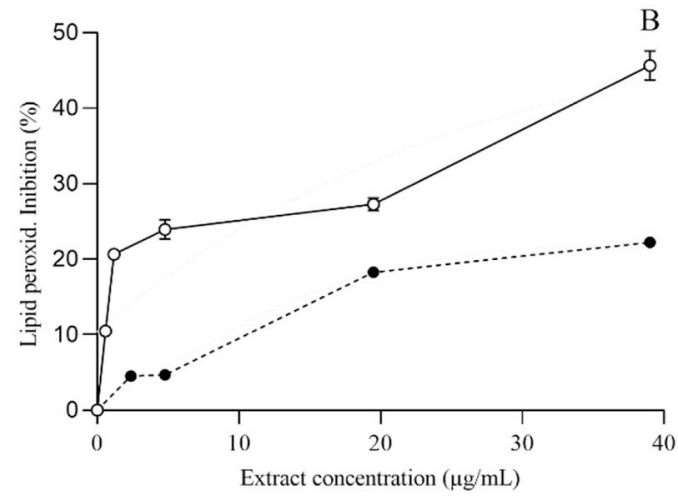

$\mathrm{D}$

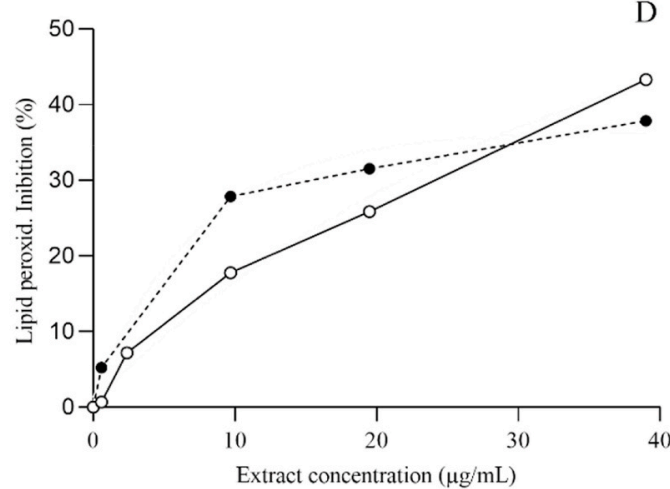

Fig. 2. Percentage of lipid peroxidation inhibition in extracts of oven-dried (-०-) and freeze-dried samples (-๑-) of $S$. minor from different origins. EC ${ }_{50}$ : extract concentration corresponding to half of lipid peroxidation inhibition. Trolox standard ( $\mathrm{EC}_{50}$ values): $0.17 \pm 0.01 \mu \mathrm{g} / \mathrm{mL}$. Oven-dried samples: $\mathrm{EC}_{50}=0.005 \pm 0.0002$; $0.03 \pm 0.002 ; 0.005 \pm 0.0002 ; 0.03 \pm 0.0001 \mu \mathrm{g} / \mathrm{mL}$ in plant material collected as wild (A-W) or provided by three different local farms B-F1; C-F2; D-F3), respectively; freeze-dried samples: $\mathrm{EC}_{50}=0.04 \pm 0.001 ; 0.06 \pm 0.006 ; 0.02 \pm 0.003 ; 0.05 \pm 0.003 \mu \mathrm{g} / \mathrm{mL}$ in plant material collected as wild (A) or provided by three different local farms (B, C, D like F1, F2 and F3), respectively.

weakest activity was observed in FD samples, particularly in samples from $\mathrm{F} 1$ and $\mathrm{F} 3\left(\mathrm{EC}_{50} 0.06 \pm 0.006\right.$ and $0.05 \pm 0.003 \mu \mathrm{g} / \mathrm{mL}$, respectively). These results were in disagreement with some authors that reported the FD as the most effective method to preserve medicinal and food plants and their nutraceutical properties (Asami et al., 2003; Meng et al., 2018). However, these results agree with recent findings of other authors (Barroso et al., 2018; Roshanak et al., 2016) as well as with the content of phenolic compounds observed in both OD and FD samples in the present study (Table 2). Indeed, Roshanak et al. (2016), comparing seven different drying treatments (sun, shade, oven $60^{\circ} \mathrm{C}$, oven $80^{\circ} \mathrm{C}$, oven $100{ }^{\circ} \mathrm{C}$, microwave, and freeze-drying), observed the maintenance of the antioxidant activity in green tea dried in the oven at $60{ }^{\circ} \mathrm{C}$, whilst the other drying methods reported a decrease of antioxidant activity. Moreover, Barroso et al. (2016) showed higher antioxidant activity in OD Stevia rabaudiana Bertoni than in fresh frozen samples.

\subsection{Antibacterial activity}

The antibacterial activity of OD and FD $S$. minor samples was tested against strains of eight bacteria: E. coli, K. pneumoniae, M. morganii, $P$. mirabilis, $P$. aeruginosa, E. faecalis, L. monocytogenes, and MRSA and the results were expressed in Table 3. The MIC values varied from $2.5 \mathrm{mg} /$

Table 3

Antibacterial activity of oven-dried and freeze-dried samples of $S$. minor.

\begin{tabular}{|c|c|c|c|c|c|c|c|c|c|c|}
\hline & \multicolumn{4}{|c|}{ Oven-dried } & \multicolumn{4}{|c|}{ Freeze-dried } & \multirow[t]{2}{*}{ Ampicilin } & \multirow[t]{2}{*}{ Vancomicyn } \\
\hline & $\mathrm{W}$ & $\mathrm{F} 1$ & $\mathrm{~F} 2$ & F3 & $\mathrm{W}$ & $\mathrm{F} 1$ & $\mathrm{~F} 2$ & F3 & & \\
\hline \multicolumn{9}{|c|}{ Antibacterial activity $(\mathrm{mg} / \mathrm{mL})$} & \multicolumn{2}{|c|}{ Control (20 mg/mL) } \\
\hline Bacteria & MIC & MIC & MIC & MIC & MIC & MIC & MIC & MIC & MIC & MIC \\
\hline \multicolumn{11}{|l|}{ Gram-negative } \\
\hline Escherichia coli & 0.5 & 5 & 5 & 10 & 5 & 5 & 5 & 10 & $<0.15$ & nt \\
\hline Klebsiella pneumoniae & 5 & 10 & 10 & 10 & 10 & 20 & 20 & 10 & 10 & nt \\
\hline Morganella morganii & 5 & 10 & 10 & 10 & 10 & 10 & 10 & 10 & 20 & nt \\
\hline Proteus mirabilis & 10 & 10 & 10 & 20 & 10 & 20 & $>20$ & 20 & $<0.15$ & nt \\
\hline \multicolumn{11}{|l|}{ Gram-positive } \\
\hline Enterococcus faecalis & 5 & 5 & 10 & 10 & 5 & 5 & 5 & 10 & $<0.15$ & $<0.0078$ \\
\hline Listeria monocytogenes & 5 & 5 & 10 & 10 & 20 & 10 & 10 & 10 & $<0.15$ & nt \\
\hline MRSA & 2.5 & 5 & 5 & 20 & 5 & 10 & 5 & 20 & $<0.15$ & 0.25 \\
\hline
\end{tabular}

MIC - minimal inhibitory concentration; MRSA-methycilin resistant S. aureus; nt: not tested; W-wild-collected samples; F1, F2, F3-samples provided by local farmers. 
$\mathrm{mL}$ to $>20 \mathrm{mg} / \mathrm{mL}$ in OD and FD $S$. minor samples. The MBC values were not possible to obtain since the maximum concentration of $20 \mathrm{mg} / \mathrm{mL}$ did not present bactericidal activity. All the plant extracts revealed antibacterial activity independently to the growing technique (wild or cultivated), showing higher antibacterial activity in OD samples compared to that of FD samples in most cases. Moreover, each OD wild or cultivated exhibited a higher ability to inhibit the growth of different bacteria if compared with FD samples and, in particular, OD W plants exhibited higher inhibition of E. coli, K. pneumonia, M. morganii, $L$. monocytogenes, and MRSA growth than FD W plants. OD F1 plants showed higher activity against $K$. pneumoniae, P. mirabilis, L. monocytogenes, and MRSA, OD F2 plants against $K$. pneumoniae, $P$. mirabilis and $P$. aeruginosa and OD F3 plants against $K$. pneumoniae, $P$. mirabilis, $P$. aeruginosa, E. faecalis and L. monocytogenes than FD F1, F2, F3 samples, respectively. The only exception was the higher antibacterial activity showed by FD F2 plants towards the $E$. faecalis than the OD F2 plants. In general, the more sensitive bacteria to $S$. minor was $E$. coli among Gramnegative and MRSA among Gram-positive bacteria.

In contrast, the most resistant bacteria proved to be $P$. mirabilis and L. monocytogenes. The high antibacterial activity of $S$. minor was confirmed by other authors, even though with lower MIC and MBC values (Finimundy et al., 2020; Karkanis et al., 2019). Karkanis et al. (2019) observed MIC and MBC values of domesticated $S$. minor samples, against Bacillus cereus, S. aureus, L. monocytogenes and Salmonella typhimurium $(0.075-0.45 \mathrm{mg} / \mathrm{mL}$ and $0.25-0.60 \mathrm{mg} / \mathrm{mL}$, respectively). Beside, Finimundy et al. (2020) reported MIC and MBC values between 2.31 and $0.44 \mathrm{mg} / \mathrm{mL}$; and between 4.61 and $0.88 \mathrm{mg} / \mathrm{mL}$, respectively, against $S$. aureus, B. cereus, $M$. flavus, $L$. monocytogenes, $P$. aeruginosa, $S$. typhimurium, E. cloacae, and E. coli, analysing cultivated $S$. minor plants under different fertilization regimes. Despite the achievement of a lower antimicrobial activity than that previously reported by other authors, the present results corroborated $S$. minor species and highlighted its potential as a functional ingredient, especially if subjected to the OD method as the most effective drying technique.

\subsection{Cytotoxic and anti-inflammatory activities}

The results from the cytotoxic and anti-inflammatory activities of OD and FD S. minor samples are presented in Table 4. Regarding the cytotoxic activity, $\mathrm{OD} W$ samples exhibited $\mathrm{EC}_{50}$ values ranging from $230 \pm$ 1 to $305 \pm 7 \mu \mathrm{g} / \mathrm{mL}$, and FD W samples from $263 \pm 6$ to $327 \pm 8 \mu \mathrm{g} / \mathrm{mL}$. Both OD and FD W samples presented high activity against the HeLa cell line. Regarding OD samples, the lowest growth inhibition was observed in HepG2 and MCF-7 cell lines that reported $305 \pm 7$ and $301 \pm 7 \mu \mathrm{g} /$ $\mathrm{mL}$, respectively. On the other hand, the FD samples confirmed the lowest growth inhibition of HepG2 cell line (up to $400 \mu \mathrm{g} / \mathrm{mL}$ ), not showing hepatotoxicity to this cell line. However, wild extracts revealed the most potent capacity to inhibit the growth of the studied tumor cell lines. Among the domesticated samples, none of the extracts presented cytotoxic activity, except for the samples from F3 OD and F2 FD that revealed cytotoxicity against HeLa cells in a concentration of $225 \pm 10$ and $343 \pm 5 \mu \mathrm{g} / \mathrm{mL}$, respectively. The results of this study resulted in agreement with other authors (Finimundy et al., 2020; Karkanis et al., 2019). Karkanis et al. (2019) observed that the extracts from $S$. minor roots showed the highest activity against HeLa $\left(\mathrm{GI}_{50}=75 \mu \mathrm{g} / \mathrm{mL}\right)$. Similarly, Finimundy et al. (2020) reported the highest activity against HeLa $\left(\mathrm{GI}_{50}\right.$ range from $55 \pm 6$ to $99 \pm 5 \mu \mathrm{g} / \mathrm{mL}$ ) from $S$. minor leaves and roots subjected to different fertiliser regimes. Conversely, the weakest activity was verified against NCl-H460 (GI 50 range from $65 \pm 6$ to 293 $\pm 21 \mu \mathrm{g} / \mathrm{mL}$ ), confirming our results and suggesting that the growing conditions may affect the cytotoxic activity. Regarding anti-inflammatory activity, to the best of our knowledge, only few reports are available in the literature reporting the activity of $S$. minor. According to the obtained results, the analysed extracts did not present activity at the maximum tested concentration $(400 \mu \mathrm{g} / \mathrm{mL})$ (Table 3$)$. In contrast, Su et al. (2018) suggested that isolated compounds from the S. officinalis exhibited anti-inflammatory activity $(20 \mu \mathrm{M})$, and methanol extract $(40 \mu \mathrm{g} / \mathrm{mL})$ decreased the production of TNF- $\alpha$ in LPS-activated RAW264.7. Arihan et al. (2015) utilised aqueous $S$. minor (maceration) extracts and obtained anti-inflammatory activity at $25 \mathrm{mg} / \mathrm{kg}$ in rats.

\section{Conclusions}

A renewed interest in $S$. minor species has been showed by scientific literature for its edibility and its nutraceutical properties due to the high content in phenolic compounds. However, appropriate methods must be applied to preserve the nutraceutical properties of this species during the storage. The present study showed that OD samples presented a higher antioxidant and antimicrobial activity and a higher content in phenolic compounds when compared with FD samples, showing that the OD resulted the most indicated drying method to preserve and retain these bioactive compounds. Moreover, some differences were observed between wild and domesticated samples. Wild samples showed higher antimicrobial and cytotoxic activity than domesticated samples. Indeed, the domesticated samples did not report cytotoxic activity against the analysed cell lines, except for the OD F3 and FD F2 samples that revealed cytotoxicity against HeLa cells in a concentration of $225 \pm 10$ and $343 \pm$ $5 \mu \mathrm{g} / \mathrm{mL}$, respectively. Further researches are necessary to standardise the domestications and to verify the highest efficiency of OD storage of $S$. minor species.

\section{CRediT authorship contribution statement}

Costanza Ceccanti: Methodology, Investigation, Writing - original draft. Tiane C. Finimundy: Conceptualization, Methodology, Writing original draft. Sandrina A. Heleno: Conceptualization, Methodology,

Table 4

Anti-inflammatory and cytotoxic activity of oven-dried and freeze-dried samples of $S$. minor.

\begin{tabular}{|c|c|c|c|c|c|c|c|c|}
\hline & \multicolumn{4}{|c|}{ Oven-dried } & \multicolumn{4}{|c|}{ Freeze-dried } \\
\hline & $\mathrm{W}$ & $\mathrm{F} 1$ & $\mathrm{~F} 2$ & F3 & $\mathrm{W}$ & F1 & $\mathrm{F} 2$ & F3 \\
\hline \multicolumn{9}{|c|}{ Anti-inflammatory activity $\left(\mathrm{EC}_{50}, \mu \mathrm{g} / \mathrm{mL}\right)$} \\
\hline RAW 246.7 & $>400$ & $>400$ & $>400$ & $>400$ & $>400$ & $>400$ & $>400$ & $>400$ \\
\hline \multicolumn{9}{|c|}{ Cytotoxic activity $\left(\mathrm{GI}_{50}, \mu \mathrm{g} / \mathrm{mL}\right)$} \\
\hline MCF-7 & $301 \pm 7^{\mathrm{a}}$ & $>400$ & $>400$ & $>400$ & $323 \pm 9^{\mathrm{a}}$ & $>400$ & $>400$ & $>400$ \\
\hline NCI-H460 & $252 \pm 4^{\mathrm{a}}$ & $>400$ & $>400$ & $>400$ & $327 \pm 8^{\mathrm{b}}$ & $>400$ & $>400$ & $>400$ \\
\hline HeLa & $230 \pm 1^{\mathrm{a}}$ & $>400$ & $>400$ & $225 \pm 10^{\mathrm{a}}$ & $263 \pm 6^{b}$ & $>400$ & $343 \pm 5^{c}$ & $>400$ \\
\hline HepG2 & $305 \pm 7^{\mathrm{a}}$ & $>400$ & $>400$ & $>400$ & $>400$ & $>400$ & $>400$ & $>400$ \\
\hline PLP & $>400$ & $>400$ & $>400$ & $>400$ & $>400$ & $>400$ & $>400$ & $>400$ \\
\hline
\end{tabular}

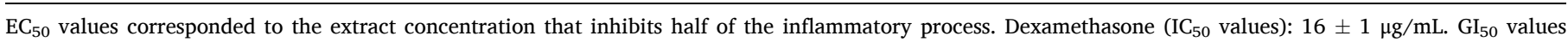

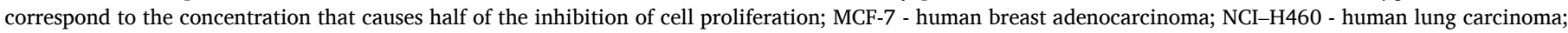

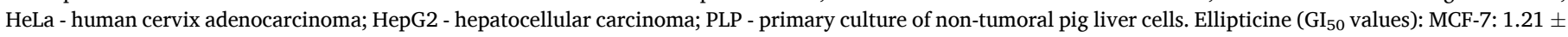

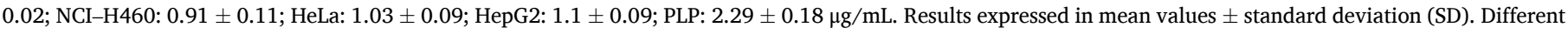
letters in the line represent significant differences $(\mathrm{p}<0.05)$. W-wild-collected samples; F1, F2, F3-samples provided by local farmers. 
Writing - review \& editing. Tania C.S.P. Pires: Methodology, Writing original draft. Ricardo C. Calhelha: Methodology. Lucia Guidi: Writing - review \& editing. Isabel C.F.R. Ferreira: Conceptualization, Methodology, Writing - review \& editing. Lillian Barros: Conceptualization, Methodology, Writing - original draft.

\section{Declaration of competing interest}

The authors declare that they have no known competing financial interests or personal relationships that could have appeared to influence the work reported in this paper.

\section{Acknowledgements}

The authors are grateful to the Foundation for Science and Technology (FCT, Portugal) and FEDER under Programme PT2020 for financial support to CIMO (UID/AGR/00690/2020). L. Barros and C. Calhelha also thank the national funding by FCT - Foundation for Science and Technology, P.I., through the institutional scientific employment program-contract for their contracts, and Sandrina A. Heleno thanks to the national funding by FCT, P.I., through the individual scientific employment program-contract. The authors are also grateful to FEDER-Interreg España-Portugal programme for financial support through the project TRANSCOLAB 0612_TRANS_CO_LAB_2_P and to European Regional Development Fund (ERDF) through the Regional Operational Program North 2020, within the scope of Project Norte-010145-FEDER-000042: GreenHealth.\

\section{Appendix A. Supplementary data}

Supplementary data to this article can be found online at https://doi. org/10.1016/j.lwt.2021.111335.

\section{References}

Alean, J., Chejne, F., \& Rojano, B. (2016). Degradation of polyphenols during the cocoa drying process. Journal of Food Engineering, 189, 99-105. https://doi.org/10.1016/j. jfoodeng.2016.05.026

Arıhan, O., Özbek, H., \& Özkan, A. M. G. (2015). Anti-inflammatory effects of Sanguisorba minor scop. subsp. muricata (Spach) Briq. And Cirsium libanoticum DC. subsp. lycaonicum (Boiss. \& Heldr.) Davis \& Parris in rat. Eastern Journal of Medicine, 20, 81-85.

Asami, D. K., Hong, Y. J., Barrett, D. M., \& Mitchell, A. E. (2003). Comparison of the total phenolic and ascorbic acid content of freeze-dried and air-dried marionberry, strawberry, and corn grown using conventional, organic, and sustainable agricultural practices. Journal of Agricultural and Food Chemistry, 51, 1237-1241. https://doi.org/10.1021/jf020635c

Barroso, M., Barros, L., Rodrigues, M.Â., et al. (2016). Stevia rebaudiana Bertoni cultivated in Portugal: A prospective study of its antioxidant potential in different conservation conditions. Industrial Crops and Products, 90, 49-55. https://doi.org/10.1016/j. indcrop.2016.06.013

Barroso, M. R., Martins, N., Barros, L., et al. (2018). Assessment of the nitrogen fertilization effect on bioactive compounds of frozen fresh and dried samples of Stevia rebaudiana Bertoni. Food Chemistry, 243, 208-213. https://doi.org/10.1016/j. foodchem.2017.09.137

Bessada, S. M. F., Barreira, J. C. M., Barros, L., et al. (2016). Phenolic profile and antioxidant activity of Coleostephus myconis (L.) Rchb.f.: An underexploited and highly disseminated species. Industrial Crops and Products, 89, 45-51. https://doi. org/10.1016/J.INDCROP.2016.04.065

Bunse, M., Lorenz, P., Stintzing, F. C., \& Kammerer, D. R. (2020). Characterization of secondary metabolites in flowers of Sanguisorba officinalis L. By HPLC-DAD-MSn and GC/MS. Chemistry and Biodiversity, 17. https://doi.org/10.1002/cbdv.201900724

Ceccanti, C., Landi, M., Incrocci, L., et al. (2020). Comparison of three domestications and wild-harvested plants for nutraceutical properties and sensory profiles in five wild edible herbs: Is domestication possible? Foods, 9, 1065. https://doi.org/ 10.3390 /foods 9081065

Ceccanti, C., Landi, M., Rocchetti, G., et al. (2019). Hydroponically grown Sanguisorba minor scop.: Effects of cut and storage on fresh-cut produce. Antioxidants, 8, 1-15. https://doi.org/10.3390/antiox8120631
Clifford, M. N., Johnston, K. L., Knight, S., \& Kuhnert, N. (2003). Hierarchical scheme for LC-MSn identification of chlorogenic acids. Journal of Agricultural and Food Chemistry, 51, 2900-2911. https://doi.org/10.1021/jf026187q

Finimundy, T. C., Karkanis, A., Fernandes, Â., et al. (2020). Bioactive properties of Sanguisorba minor L. cultivated in central Greece under different fertilization regimes. Food Chemistry, 327. https://doi.org/10.1016/j.foodchem.2020.127043

Guarrera, P. M., \& Savo, V. (2013). Perceived health properties of wild and cultivated food plants in local and popular traditions of Italy: A review. Journal of Ethnopharmacology, 146, 659-680.

Guarrera, P. M., \& Savo, V. (2016). Wild food plants used in traditional vegetable mixtures in Italy. Journal of Ethnopharmacology, 185, 202-234.

Harbourne, N., Marete, E., Jacquier, J. C., \& O'Riordan, D. (2009). Effect of drying methods on the phenolic constituents of meadowsweet (Filipendula ulmaria) and willow (Salix alba). Lebensmittel-Wissenschaft und -Technologie- Food Science and Technology, 42, 1468-1473. https://doi.org/10.1016/j.lwt.2009.05.005

Karkanis, A. C., Fernandes, A., Vaz, J., et al. (2019). Chemical composition and bioactive properties of Sanguisorba minor Scop. under Mediterranean growing conditions. Food \& Function, 10, 1340-1351. https://doi.org/10.1039/c8fo02601g

Karkanis, A., Vellios, E., Thomaidis, T., et al. (2014). Phytochemistry and biological properties of burnet weed (Sanguisorba spp.): A review. Notulae Scientia Biologicae, 6, 395-398. https://doi.org/10.1583/nsb649471

Kaufmann, D., Herrmann, F., \& Wink, M. (2009). Extracts from traditional Chinese medical plants inhibit glycogen synthase kinase $3 \beta$ activity, a potential Alzheimer target. Zeitschrift für Phytherapie, 30, V16. https://doi.org/10.1055/s-0029-1239859

Mandim, F., Dias, M. I., Pinela, J., et al. (2020). Chemical composition and in vitro biological activities of cardoon (Cynara cardunculus L. var. altilis DC.) seeds as influenced by viability: Chemical prospection and bioactivity of cardoon seeds. Food Chemistry, 323, 126838. https://doi.org/10.1016/j.foodchem.2020.126838

Meng, Q., Fan, H., Li, Y., \& Zhang, L. (2018). Effect of drying methods on physicochemical properties and antioxidant activity of Dendrobium officinale. Journal of Food Measurement and Characterization, 12, 1-10. https://doi.org/10.1007/s11694-0179611-5

Parveen, I., Winters, A., Threadgill, M. D., et al. (2008). Extraction, structural characterisation and evaluation of hydroxycinnamate esters of orchard grass (Dactylis glomerata) as substrates for polyphenol oxidase. Phytochemistry, 69, 2799-2806. https://doi.org/10.1016/j.phytochem.2008.08.019

Pinela, J., Barros, L., Dueñas, M., et al. (2012). Antioxidant activity, ascorbic acid, phenolic compounds and sugars of wild and commercial Tuberaria lignosa samples: Effects of drying and oral preparation methods. Food Chemistry, 135, 1028-1035. https://doi.org/10.1016/j.foodchem.2012.05.038

Pires, T. C. S. P., Dias, M. I., Barros, L., et al. (2018). Antioxidant and antimicrobial properties of dried Portuguese apple variety (Malus domestica Borkh. cv Bravo de Esmolfe). Food Chemistry, 240, 701-706. https://doi.org/10.1016/j. foodchem.2017.08.010

Ranfa, A., Maurizi, A., Romano, B., \& Bodesmo, M. (2014). The importance of traditional uses and nutraceutical aspects of some edible wild plants in human nutrition: The case of Umbria (central Italy). Plant Biosystems, 148, 297-306.

Roshanak, S., Rahimmalek, M., \& Goli, S. A. H. (2016). Evaluation of seven different drying treatments in respect to total flavonoid, phenolic, vitamin C content, chlorophyll, antioxidant activity and color of green tea (Camellia sinensis or C. assamica) leaves. Journal of Food Science aand Technolology, 53, 721-729. https:// doi.org/10.1007/s13197-015-2030-x

Shonte, T. T., Duodu, K. G., \& de Kock, H. L. (2020). Effect of drying methods on chemical composition and antioxidant activity of underutilized stinging nettle leaves. Heliyon, 6, Article e03938. https://doi.org/10.1016/j.heliyon.2020.e03938

Su, X. D., Guo, R. H., Li, H. X., et al. (2018). Anti-allergic inflammatory components from Sanguisorba officinalis L. Bioorganic \& Medicinal Chemistry Letters, 28, 2210-2216. https://doi.org/10.1016/J.BMCL.2018.04.033

Svobodova, B., Barros, L., Sopik, T., et al. (2017). Non-edible parts of Solanum stramoniifolium Jacq. - a new potent source of bioactive extracts rich in phenolic compounds for functional foods. Food \& Function, 8, 2013-2021. https://doi.org/ $10.1039 / \mathrm{c} 7$ fo00297a

Terefe, N. S., Yang, Y. H., Knoerzer, K., et al. (2010). High pressure and thermal inactivation kinetics of polyphenol oxidase and peroxidase in strawberry puree. Innovative Food Science \& Emerging Technologies, 11, 52-60. https://doi.org/10.1016/ j.ifset.2009.08.009

Vanzani, P., Rossetto, M., De Marco, V., et al. (2011). Wild Mediterranean plants as traditional food: A valuable source of antioxidants. Journal of Food Science, 76. https://doi.org/10.1111/j.1750-3841.2010.01949.x

Welch, R. M., \& Graham, R. D. (1999). A new paradigm for world agriculture: Meeting human needs. Productive, sustainable, nutritious. Field Crops Research, 60, 1-10. https://doi.org/10.1016/S0378-4290(98)00129-4

Ye, S., Wang, Z., Shen, J., et al. (2019). Sensory qualities, aroma components, and bioactive compounds of Anoectochilus roxburghii (Wall.) Lindl. as affected by different drying methods. Industrial Crops and Products, 134, 80-88. https://doi.org/ 10.1016/j.indcrop.2019.03.063

Youssef, K. M., \& Mokhtar, S. M. (2014). Effect of drying methods on the antioxidant capacity, color and phytochemicals of Portulaca oleracea L. Leaves. Journal of Nutrition \& Food Sciences, 4. https://doi.org/10.4172/2155-9600.1000322 